Reproduced from

FOUNDATIONS OF PHYSICS Vol. 9, pp. 803 - 818 (1979)

(with minor corrections and reformulations)

\title{
QUANTUM THEORY AND TIME ASYMMETRY
}

\author{
H. D. Zeh \\ Institut für Theoretische Physik \\ Universität Heidelberg \\ www.zeh-hd.de
}

\begin{abstract}
The relation between quantum measurement and thermodynamically irreversible processes is investigated. The reduction of the state vector is fundamentally asymmetric in time and shows an observer-relatedness which may explain the double interpretation of the state vector as a representation of physical states as well as of information about them. The concept of relevance being used in all statistical theories of irreversible thermodynamics is shown to be based on the same observer-relatedness. Quantum theories of irreversible processes implicitly use an objectivized process of state vector reduction. The conditions for the reduction are discussed, and I speculate that the final (subjective) observer system might even be carried by a spacetime point.
\end{abstract}




\section{INTRODUCTION}

Several contributions concerned with the problem of measurement in quantum theory suggested a close relationship between the measurement process and irreversible processes in statistical physics [1-4]. In fact, there are very plausible arguments supporting this idea: quantum measurements seem to require irreversible thermodynamics for the amplification of microscopic phenomena, while the consistency problems between deterministic ${ }^{1}$ equations of motion and master equations on the one hand, and between the Schrödinger equation and the statistical character of quantum measurement on the other, appear analogous in some respect. Moreover, the ensemble (incomplete information) concept of statistical physics and the popular interpretation of the wave function as representing information about physical systems both contain a similar observer-relatedness. (In contrast, the time asymmetry connected with $\mathrm{CP}$ violation is described by a unitary time evolution, and does not show any relation to the two other time-asymmetric phenomena.)

Although the formal parts of the above-cited investigations are essentially clear, there are deep differences concerning their interpretation. There are claims that these contributions serve to derive the statistical nature of quantum measurements from thermodynamical fluctuations, while in general the statistical nature of quantum theory is regarded as specific and fundamental.

Quite obviously, these discrepancies are based on different positions concerning the basic concepts of quantum theory. It is generally accepted that this theory makes statistical predictions: it describes ensembles of final states appearing in a measurement process. This statement remains incomplete as long as the conceptual terms to describe the members of these ensembles are not defined. That is, the kinematical concepts (particle positions, wave functions, or something else) to be used to describe the physical state of the apparatus after a measurement (its "pointer position" or measurement result) have to be chosen, and the same choice is required for the time before and during measurements in order to formulate a dynamical theory of measurement. A specific question is whether classical concepts are fundamentally required to describe the measurement results, or whether they are no more than a short-hand descriptions for certain quantum mechanical state vectors ("derived classical concepts"). For example, the spot

\footnotetext{
${ }^{1}$ The term "deterministic" is here used to characterize dynamical laws possessing unique solutions, although some of the founders of quantum theory interpreted this term (and correspondingly its apparent opposite: the quantum mechanical "indeterminacy") as the possibility of (in principle) completely determining the required initial conditions.
} 
resulting on a photographic plate in the measurement of a photon position can be described by a stable, localized change in the corresponding molecular wave functions, while the "classical" position of a pointer may be replaced by the sufficiently localized center-of-mass part of the many-particle wave function for the pointer. The probability interpretation of quantum theory then has to be expressed by means of a collapse (or reduction) of the state vector.

In order to avoid conceptual confusion, some basic interpretational issues of quantum theory are recalled in Section 2, before one of them is chosen for the further discussion. In Section 3, some fundamentals of statistical physics are discussed, and the role of observer-related concepts, such as relevance and information, is pointed out. Their relation to the quantum measurement process is studied in Section 4. Speculations about a possible solution of the measurement problem are presented in Section 5.

\section{CONCEPTUAL AND DYNAMICAL DUALISM}

According to Niels Bohr's epistemology, quantum mechanics is incompatible with the existence of a real microscopic world. It is meaningless to ask whether an electron "really" is a particle or a wave - each choice would lead to false conclusions. Instead, the wave function is assumed to be a tool for calculating probabilities for potential results of measurements ("pointer positions" of a macroscopic device). These have to be described by classical concepts, which, however, are subject to the uncertainty relations. During a measurement, the electron may "assume" a definite position or momentum (that is, a classical property). On the other hand, quantum mechanics is assumed to be universally valid. This would mean that the apparatus itself must possess a quantum mechanical state vector (non-relativistically represented by a many-particle wave function).

It seems that by "reality" (to be rejected for the microscopic world) Bohr meant the existence of general and consistently applicable concepts to describe states of physical objects. His "conceptual dualism" between classical and quantum concepts goes beyond the dualism characterized by conjugate variables, such as position and momentum, or particle number and field.

According to another interpretation - I shall call it von Neumann's the state vector is regarded as generally and exclusively applicable if in addition to the Schrödinger equation another dynamical law (its "collapse" or "reduction") is assumed to apply. This collapse describes an unpredictable 
transition of a general state vector into one of its components of a certain representation,

$$
\sum_{l} c_{l} \psi_{l} \rightarrow \psi_{l}
$$

This existence of two different dynamical laws may be called a "dynamical dualism". The reduction is indeterministic: the state vector allows us to predict only an ensemble of potential state vectors at later times. In this way, the state vector itself represents an objective physical state, but it contains only incomplete information about the future (or past!) state. There are only intuitive rules to decide which one of the two dynamical laws to use in a certain situation. By definition, the reduction applies in "measurementlike" situations, where the final states are eigenstates of phenomenologically chosen observables. ${ }^{2}$

The reduction (that is, the dynamical dualism) has to be used also in Bohr's interpretation, in particular if a measurement serves to prepare initial states for a second one. Intuitive rules are then again required to switch between classical variables and quantum mechanical state vectors.

Both interpretations appear useful pragmatically. Von Neumann's interpretation may be preferable for fundamental discussions, since it is at least conceptually unambiguous. It does not presume any classical concepts, except for constructing the specific state space of a quantum system whose classical description is known beforehand. For example, an individual droplet in a Wilson chamber would not be described simply by its classical position, but instead by a localized wave packet for the center of mass of an aggregate of molecules. Moreover, it seems to be possible to decide on purely dynamical grounds [5] which properties behave classically under specified circumstances. For example, particle aspects are preferred for charged fermions under "normal" conditions, but wave aspects for most electromagnetic phenomena or for other bosons under conditions of superfluidity. In general, the quantum state of a certain space region (which may include the apparatus and/or the observer) may according to quantum field theory not only be found in classical field configurations, but also in superpositions thereof.

A state vector (such as represented by a many-particle wave function) is usually rejected as describing an individual physical state, since it is a

\footnotetext{
${ }^{2}$ So-called measurements of the second kind, for which the collapse component $\psi_{l}$ does not correspond to the eigenstate of the measured quantity, can be reduced to measurements of the first kind by enlarging the considered system appropriately (for example, to include the measurement apparatus): the "pointer" always remains in its observed state directly after being read.
} 
nonlocal concept [6]. This nonlocality of quantum theory has well known consequences $[7,8]$, and it is a direct consequence of the superposition principle. It is compatible with the dynamical locality presumed in field theories or used in non-relativistic many-particle wave mechanics with contact interactions. The kinematical nonlocality is at the heart of the EPR problem $[9,10]$, while its consequences have recently been experimentally verified over macroscopic distances [11] by using Bell's inequality [12]. Bell's analysis has clearly revealed that any realistic theory (for example in the sense of Einstein, Podolsky, and Rosen [9]) which is experimentally equivalent to quantum theory must necessarily be nonlocal. This could only be avoided if the hidden variables behaved in a thermodynamically unusual way (conspiratorially or teleologically, that is, according to special final conditions). Disregarding this latter possibility, there is thus no good reason to reject the state vector as representing reality.

Since the state vector is a nonlocal concept, it cannot generally be applied to local systems. (A local system may be defined by means of a time-like world tube in four-dimensional spacetime.) For dynamical reasons, no state vector can ever be consistently applied to a macroscopic system, except for the universe as a whole $[13,14]$, and only under special circumstances does it even apply to a microscopic system. Hence, the reduction cannot be ascribed to perturbations caused by the observer or the environment, since the state vector used in Eq. (1) must be assumed to represent the whole universe, thus already including all possible perturbations.

The dynamical dualism may be avoided in the interpretation of quantum theory proposed by Everett [15]. The existence of many unobserved world components - postulated in this interpretation - is usually regarded as an unnecessary and extravagant complication. The assumption of "other" components (which would disappear according to the reduction) is in fact as unnecessary, but also as natural, as the assumption of the existence of objects while not being observed: it follows from an extrapolation of the empirical laws of nature (in Everett's case the Schrödinger equation). However, the description of our observed universe in derived classical terms corresponds to the reduced states, wherein classical properties appear as wave packets that are approximate eigenstates of the phenomenological observables. Therefore, von Neumann's interpretation will be preferred to Everett's in what follows, except where explicitly stated. As will be discussed in Section 5, Everett's "branching" is based on a similar observer-relatedness as von Neumann's reduction.

The problem of quantum measurement thus concerns the dynamics of the state vector during a measurement (the reduction). Bohr's interpretation of 
the wave function would not even offer concepts for a non-phenomenological description of measurements. It refers to an outside observer - in conflict with quantum nonlocality [14]. The ensemble of potential collapsed state vectors (formally represented by a density matrix lacking the initial interference terms) can evolve from the original state vector only by means of an indeterministic law. Just one member of this ensemble - the observed one - may then be considered as "real" (although this is a matter of definition, as can be seen from Everett's interpretation). In contrast, the ensemble describing all potential results characterizes the incomplete predictability according to this indeterminism. The remaining questions then are: (a) When precisely does the reduction apply instead of the Schrödinger equation? (b) Which interference terms disappear in a certain situation, i.e., into which components does the total state vector collapse (or, equivalently, how to justify the phenomenological observable)?

\section{THE CONCEPT OF RELEVANCE IN STATISTICAL PHYSICS}

Statistical theories of irreversible thermodynamics are fundamentally based on a concept of "relevance" (generalized coarse-graining) and certain initial (rather than final) conditions. The latter may be special for the relevant variables, but random with respect to the irrelevant ones. Statistical methods may then be used to derive master equations which approximately describe the dynamics of the relevant quantities. The latter are often regarded as macroscopic, and will be shown to be related to the classical concepts in quantum theory. Master equations are able to describe the arrow of time experienced by us as causality and an apparently "fixed past". Since this is usually regarded as the reason why initial conditions may be "given", this argument may appear circular from a fundamental point of view.

Master equations are based on various appropriately chosen definitions of relevance. Examples of quantities considered as irrelevant are particle correlations in Boltzmann's H-theorem, fine-grained phase-space positions in Gibbs' ink drop analogy, or certain phase relations in quantum theories of irreversible processes. Zwanzig [16] formalized the general concept of relevance by means of projection operators $P$ in the space of phase-space densities $\rho(p, q)$ (in classical mechanics) or density matrices $\rho_{m n}$ (in quantum mechanics). He derived a general dynamical description (pre-master equation) of the projected densities $\rho_{\text {rel }}=P \rho$ by methods which had been developed for special projections by van Hove [17] and Prigogine [18], and 
he demonstrated that most examples studied by the great pioneers of statistical physics could be formulated in terms of the projection method. It was conceptually only of secondary importance that Zwanzig's procedure turned out to be too general on the one hand (since not all projections of density matrices are density matrices again) and too limited on the other (as many important examples of relevance have to be described by nonlinear idempotent operators $[19,20]$. In the following, the term "Zwanzig projection" is used for all appropriate idempotent operations.

The concept of relevance introduces an observer-related element. This appears incompatible with our general understanding that the laws of thermodynamics are objective. The Zwanzig projection is able to map pure ("real") states into ("representative") ensembles. The latter seem to correspond to the observer-related concept of incomplete information, where entropy appears as a measure of lacking information. In contrast to information theory, some objectivization is obtained in statistical physics by calculating the entropy not from the actual information, but by assuming that the relevant (or "easily accessible") quantities are always known, while the irrelevant ones are never known and equally distributed with respect to a certain measure. For example, long-range order parameters always appear easily accessible; this may explain the relation between entropy and disorder. Nevertheless, the concept of relevance is fundamentally subjective. From an objective point of view there is no reason why the position of an individual molecule should be irrelevant, and even for practical applications may the value of entropy depend upon whether one decides to consider fluctuations or not. Although relevance also depends on objective properties - a quantity must be regarded as relevant if it can easily influence other relevant quantities -, there is a conceptual chain of relevant quantities that can only end with the (potential) observer. It must be expected that at the end of this chain (in the observer's brain) microscopic properties are relevant again. Objective criteria by themselves, such as dynamical stability, are certainly not sufficient, as is demonstrated by the general (even classically nonlocal) constants of motion, which are quite irrelevant in the sense of statistical mechanics.

Because of the dynamical coupling between relevant and irrelevant quantities, described by Zwanzig's reversible and still exact pre-master equation

$$
\begin{aligned}
i \rho_{r e l}(t)=\quad & P L \rho_{r e l}(t)+P L \mathrm{e}^{[-\mathrm{i}(1-P) L t]} \rho_{i r r}(0) \\
& -\mathrm{i} \int d \tau P L \mathrm{e}^{[-\mathrm{i}(1-P) L \tau]}(1-P) L \rho_{r e l}(t-\tau),
\end{aligned}
$$

where $\mathrm{L}$ is the Liouville operator, $L \rho=[H, \rho]$, entropy as a measure of the 
(by definition missing) irrelevant information need not be constant in time under deterministic equations of motion. Total entropy may be defined as ${ }^{3}$

$$
S:=-k \operatorname{tr}\left(\rho_{r e l} \ln \rho_{r e l}\right)=-k \int d \alpha w_{\alpha} \ln w_{\alpha}+\int d a w_{\alpha} S_{\alpha},
$$

where $\alpha$ denotes the values of the relevant quantities, while $w_{\alpha}=\operatorname{tr}\left(P_{\alpha} \rho\right)$, and $P_{\alpha}$ is the projector onto the subspace corresponding to $\alpha$. The first term on the RHS describes the entropy of any lacking relevant information, while the second one averages accordingly over the "physical" (objectivized) entropies $S_{\alpha}:=-k \operatorname{tr}\left(\rho_{\alpha} \ln \rho_{\alpha}\right)$ as a function of $\alpha$, where $\rho_{\alpha}:=P_{\alpha} / \operatorname{tr} P_{\alpha}$ is independent of the "subjective" $\rho$. However, there is no reason for a monotonic increase of entropy without an appropriate assumption about initial conditions. Thereafter, it is a very plausible consequence of the large number of degrees of freedom (although hard to prove in general, because of the existence of singular counterexamples) that the entropy of closed systems will in practice never decrease any more. Furthermore, in many cases the feedback from irrelevant information into relevant one may completely or partially be neglected because of the large information capacity defined by the irrelevant degrees of freedom (related to their enormous Poincaré cycles). This leads to the justification of general master equations in the form

$$
\dot{\rho}_{r e l}(t)=-P L \int_{0}^{\infty} d \tau \mathrm{e}^{[-i(1-P) L \tau]}(1-P) L \rho_{\text {rel }}(t)
$$

They describe the effective dynamics of the relevant degrees of freedom for states in accordance with the special initial conditions. Master equations are equivalent to an alternating application (in the "forward" direction of time) of deterministic dynamics and Zwanzig projections [22], whereby the latter are responsible for the increase of entropy.

The remaining fundamental problem is to understand the origin of the special initial conditions. By applying statistical arguments to them, one would expect the relevant quantities always - even initially - to be close to their equilibrium values. The special conditions cannot simply be explained as being due to the preparation, because the process of preparation is just an example of interacting physical systems; the preparator himself is prepared by his environment. The initial conditions thus have to be considered as of cosmological origin. This demonstrates how the concepts of relevance are extrapolated beyond the subject's existence after having been objectivized. It is certainly a nontrivial fact that the universe is special in its initial

\footnotetext{
${ }^{3}$ See Ref. 21 for formal properties resulting for various definitions of entropy.
} 
conditions, and therefore asymmetric in time, and it appears difficult to justify a concept of relevance in purely objective terms. However, it would be most remarkable if the world were asymmetric in terms of precisely those variables that are relevant to an observer who later evolves as an effect of these special initial conditions.

Zwanzig's method of describing the dynamics of relevant properties under the assumption of special initial conditions seems to be completely analogous for classical and quantum physics. However, this is only true for the formalism - not for its interpretation.

The classical probability density $\rho(p, q)$ uniquely describes an ensemble of states (points in phase space). One assumes, in this case, that one of these points represents reality. The observer may then "pick out" a subensemble by a non-disturbing observation (increase of his information). All previous observations (his own and others'), which must also have reduced the initial ensemble, have to be compatible with this final subensemble. Physically, the observation means that the observer interacts with the system in such a way that some of his variables change in dependence of the system's variables which distinguish between these subensembles. The "sensitivity" of the observer to these variables finally determines what is relevant. This observation process should be discussed in microscopic terms. In order to avoid a subsequent reversal of this observation, an in practice irreversible process of information storage (in the sense of very long Poincaré cycles) must accompany the observation. In phenomenological terms one should expect the observer system to serve as an entropy sink [23] in order to compensate for the entropy decrease corresponding to the reduction of the ensemble. ${ }^{4}$ Classically, there is no limit for the capacity of such a sink, since the phase space of continuous observer variables could be arbitrarily fine-grained, and their initial entropy has no lower bound.

This actual information gain by an observer has to be distinguished from a process which seems to describe a decrease in objectivized entropy. To illustrate: if droplets condense out of an undercooled gas, their shape and position may be regarded as macroscopic (choice of a relevance concept), and therefore as "given" - regardless of an actual observation process. The condensation into definite droplets thus seems to describe a decrease of physical entropy, equivalent to the measure of information describing the positions and shapes. However, if the transition from one droplet position into another

\footnotetext{
${ }^{4}$ Brillouin [24] argued that this entropy decrease is compensated for by an entropy increase in the communication medium (for example, light). This is in general true, but one can in principle think of a direct interaction between system and observer.
} 
one - except for their collective continuous motion - is possible only through evaporation and re-condensation, condensation would only then form an irreversible process if the number of micro-states for each droplet position is larger than the corresponding number of micro-states for the uncondensed gas. The entropy increase during the condensation process into the ensemble of unknown droplet positions must therefore at least compensate the increase of information during a subsequent observation of the droplet position. A similarly rigorous argument cannot be found for Szillard's process of an actual-information gain, although accompanying processes will always by far overcompensate it.

\section{STATE VECTOR REDUCTION IN QUANTUM STATISTICAL PHYSICS}

In contrast to the classical probability density $\rho(p, q)$, the quantum mechanical density matrix $\rho_{m n}$ does not uniquely correspond to an ensemble of states. Although it is often formally represented by the ensemble of orthogonal states $\phi_{i}$ which diagonalize it (in fact, the entropy is calculated from this ensemble), the density matrix defines nonvanishing probabilities also for components of these states. This is true because of the fundamental probability postulate of quantum theory, which states that a state $\chi$ may be found in another state $\phi$ (if $\langle\phi \mid \chi\rangle \neq 0$ ) in a measurement. Only because of this postulate can different ensembles of not necessarily mutually orthogonal states be equivalent and be represented by one and the same density matrix $[25]$.

For these reasons, the process of observation can quantum-mechanically not be described in perfect analogy to the classical process of "picking out". It is true that an element of a given ensemble of wave functions $\left\{\phi_{i}\right\}$ could be picked out by means of an interaction of $\phi_{i} \Phi \rightarrow \phi_{i} \Phi^{(i)}$, where $\Phi$ describes the observer. The observer state would then change in dependence on the state of the observed system, and the observer becomes aware of the property $i$. However, there are also observations of a superposition $\sum c_{i} \phi_{i}$ by means of the same interaction. They are described by the dynamical process

$$
\sum_{i} c_{i} \phi_{i} \Phi_{0} \rightarrow \sum_{i} c_{i} \phi_{i} \Phi^{(i)} \rightarrow \phi_{i} \Phi^{(i)}
$$

This process contains a reduction of the wave function as the second step. The reduction changes the state vector - it does not merely describe the pickout of some pre-existing subensemble - yet it cannot be described by the 
Schrödinger equation [26]. This conclusion holds no matter how complicated the system $\Phi$, which contains the observer, may be. ${ }^{5}$

If one attempts, in analogy to the classical case, to introduce an objectivized concept of relevance, and to define the entropy as a function of the relevant quantities, one presumes that the latter are always given for the real physical state. That is, one assumes that the corresponding reductions always occur (without any measurement). Since this assumption excludes conjugate measurements, it is equivalent to the introduction of superselection rules. Quantum mechanical master equations based on a relevance concept (a Zwanzig projection) - for example, by introducing a restricted set of observabIes corresponding to a reducible algebra [27] - therefore presuppose the reduction process. This reduction is thus responsible for their nonunitarity. It is then not surprising that the master equation can be used (in a vicious circle) to "derive" the reduction, thus erroneously indicating that an irreversible amplification of fluctuations in the classical sense is the true cause of the indeterminacy of quantum measurements. (The microscopic degrees of freedom of the apparatus can not be the hidden variables which would determine the measurement outcome.)

This objectivization of the reduction beyond measurements proper illustrates the possibility (to be discussed further in Section 5) to assume that the reduction in a measurement occurs as soon as the measured superposition has been amplified to the macroscopic scale. This seems to be the reason why the objective existence of classical properties has become part of our intuition. Conversely, this impossibility of in practice distinguishing the superposition from an ensemble after a measurement leads to the consequence that the reduction (or an Everett branching) can be confirmed only by the final subjective observation - in close relationship to the application of chains of relevance ending at the observer in classical statistical physics. The relation between a reduction and subjective awareness - suspicious to most physicists as investigators of an objective reality - appears to some $[28,29]$ as the most consistent interpretation, since (a) deviations from the Schrödinger equation never had to be used except for measurement-like processes, and (b) fundamentally new laws may be expected in connection with

\footnotetext{
${ }^{5}$ Since a macroscopic observer can never be kinematically isolated in quantum theory [14], he has to be considered as an open system. Pragmatic theories of open systems are phenomenological and approximate descriptions of their interactions with the environment, similar to master equations. In order to write down a non-phenomenological theory, one has to make the "weak quantum cosmological assumption" that there is a state vector for the universe such that $\Phi$ in (5) describes the "rest of the universe", including the observer.
} 
fundamentally new concepts, such as awareness, which transcends physics but is obviously coupled to physics by physical processes of observation.

Our conventional concept of an evolving state of the universe in terms of derived classical terms makes permanent use of the reduction as an indeterministic and symmetry-violating process. The initial state of the universe may have been completely symmetric; the reduction - no matter when and where precisely it occurs - would create the complexity of the world by its symmetry-breaking power [30]. ${ }^{6}$ It forces "relevant" properties to assume definite values by projecting the state vector onto their corresponding subspaces. Although precise rules for the reduction have never been given, these rules should confine and help to define the physical meaning of relevance. The essential lesson of quantum theory is that the conventional (classical) physical reality cannot be assumed to be independent of the fundamental concepts of relevance and reduction.

\section{THE PHYSICAL EVENT OF OBSERVATION}

A phenomenon is "observed" when an observer becomes aware of it. This requires the observed system to affect the ultimate observer system, which is known to be localized in the brain and probably in the cerebral cortex. This description is utterly nontrivial in quantum theory, since a state of this system cannot even exist because of quantum nonlocality. The state of awareness or consciousness can therefore not simply correspond (in the sense of a naive psycho-physical parallelism) to "the" state of the observer system. How can local awareness be related to drastically nonlocal physical concepts?

The state of the final observer system (or at least its essential variables) is relevant in an absolute (though subjective) sense. Objectivized concepts of relevance and observation (for example, by an apparatus or the human sensorium) are derived and of secondary nature for this purpose.

Although a quantum mechanical state vector is nonlocal, that is, not in general defined in terms of any state vectors of its subsystems, quantum theory is special among nonlocal theories. Potential state vectors for subsystems are defined, while the general state of the total system can be expanded in terms of direct products of them as a consequence of the superposition principle.

Although the observer system is a subsystem of the universe, its relevant

\footnotetext{
${ }^{6}$ The expectation expressed in Ref. 30 that superpositions of different classical vacua may not exist does not seem to be generally justified.
} 
properties must be "given" to the subjective observer whenever he is aware of them. In quantum mechanical terms, this may be achieved by a reduction of the state vector. Definite relevant properties of the ultimate observer system are a minimum requirement for the mechanism of the reduction (if there really is one to explain definite observations). While one may assume that the derived and objectivized (classical) relevant properties, too, assume definite values by means of the reduction [31,32], there can be no reduction, in general, for microscopic properties (such as electron positions). The borderline between quantum and classical description had repeatedly to be shifted toward the observer - far beyond the microscopic realm whenever new experimental techniques allowed the observation of quantum mechanical phase relations. This is well demonstrated by superconductivity and other long-range phenomena of quantum coherence, but most rigorously by the quantum nonlocality experiments [11].

These considerations tend to show that in quantum theory the reduction (true or apparent) is responsible for the fact that relevant (and therefore also classical) properties may be "given". Reductions may irreversibly create initial conditions - they don't merely select them [25,30]. Anthropocentric reasons may be required in addition [33] to explain the special conditions describing "our" universe, that is, our special world branch that has resulted from all those reductions which must have occurred in the past.

Is it possible to further constrain the mechanism of the reduction? A speculative proposal will now be presented. If there does exist a psychophysical coupling, it should be formulated in most fundamental physical terms. Therefore, it should use strictly quantum mechanical concepts and remain compatible with the principles of relativity. This novel parallelism (based on reduced state vectors or, alternatively, Everett's "relative states") seems to form the main difference between classical and quantum theories.

The physical counterpart of consciousness appears to be local. Let this local system be represented in terms of an orthonormal basis of states $\phi_{i}$. If $\psi_{k}$ is a basis of states for the rest of the universe, a general global state can be written as $\sum_{i k} \phi_{i} \psi_{k}$. According to the minimum requirement for the reduction mentioned above, the reduced state after observing a result $l$, say, would be a product state, $\hat{\phi}_{l} \hat{\psi}_{l}$, if $\phi$ describes only the ultimately relevant degrees of freedom. Quite generally, the reduction is asymmetric in time, since it (indeterministically) transforms an entangled state at time $t-\epsilon$ into a product state at $t+\epsilon$. If entropy is defined to be additive (an extensive quantity) by choosing a local concept of objectivized relevance, the reduction lowers this entropy. A general theory for the mechanism of the reduction would have to explain or define (a) the observer system, (b) the basis of 
states $\hat{\phi}_{l}$, and (c) the time for the occurrence of a reduction (if any).

We know that the space of states possesses an inner product, and that the different states $\hat{\psi}_{l}$, observed in a certain measurement, are mutually orthogonal with respect to this inner product. The factor states can then be defined unambiguously (except for degeneracy) $[34,35]$ by the plausible requirement that also the corresponding observer states $\hat{\phi}_{l}$ are orthogonal, so that the total state can be written as the single sum,

$$
\sum_{i k} c_{i k} \phi_{i} \psi_{k}=\sum_{l} \hat{c}_{l} \hat{\phi}_{l} \hat{\psi}_{l}
$$

The transition from the state vector (6) to the ensemble of product states $\hat{\phi}_{l} \hat{\psi}_{l}$ with probabilities $\left|c_{l}\right|^{2}$ would then be described by the Zwanzig projection corresponding to the neglect of quantum correlations (entanglement) between the two subsystems. This hypothesis would answer question (b), provided the observer system (question (a)) were given.

We also know empirically that the observer system is spatially bounded (although we cannot give its precise boundaries), and that consciousness changes with time. If consciousness is in fact defined (and different) at every moment of time, it should also be related to points in space: the truly subjective observer system should be related to spacetime points [36]. This hypothesis may be supported by the "holographic picture" of the brain, or by Sperry's split-brain experiments [37]. One would not even be in conflict with empirical evidence when assuming that every spacetime point carries consciousness: we can only communicate with some of them, and with other brains only as a whole, in a nontrivial manner. The identity in time of the subjective "I" appears as no more than a pragmatic concept, resulting from strong causal relationships. Only the subjective "I-and-Now" is required as a fundamental concept.

Before investigating the physical consequences of this hypothesis further, briefly consider question (c). This and some of the following discussion is not specifically based on the radical hypothesis of point-like observer systems.

There is no natural time interval between reductions. This difficulty is hard to overcome. Therefore, it appears fortunate that Everett's interpretation, which does not require the reduction, is in practice equivalent to von Neumann's. One may then apply Erhard Schmidt's canonical representation (6) to the never reduced state vector at every moment of time in order to postulate [14] that consciousness is "parallel" to one of the states $\hat{\phi}_{l}$ (or to all of them separately). ${ }^{7}$ This new hypothesis avoids the dynamical time

\footnotetext{
${ }^{7}$ The branching with respect to different "memory states", proposed by Everett, would correspond to an objectivized reduction.
} 
asymmetry of the reduction. As discussed in a previous paper [38], Everett's model (which corresponds to a pre-master equation) is indistinguishable in practice from von Neumann's interpretation only if all unobserved (nonexisting in von Neumann's interpretation) components $l^{\prime} \neq l$ which might later interfere with the observed one possess negligible amplitudes $c_{l^{\prime}}$. In this way, the time-asymmetric dynamical law of reduction is replaced by an asymmetric assumption about cosmological initial conditions for the total Everett wave function [36]. The space of unobserved Everett branches has to be sufficiently empty in order to serve as a perfect sink for initially local quantum phases. (One may have to use quantum gravity in order to decide if this emptiness is related to the black night sky or the thermodynamical arrow of time $[39,40]$.)

Consider a space-like hypersurface of spacetime described by a constant time coordinate $t$, and assume that the general state vector $|\alpha(t)\rangle$ on this hypersurface can be written as a wave functional $\Psi\left[\phi_{\nu}(\mathbf{x}), t\right]$,

$$
|\alpha(t)\rangle=\int \mathcal{D} \phi_{\nu}(\mathbf{x}) \Psi\left[\phi_{\nu}(\mathbf{x}), t\right]\left|\phi_{\nu}(\mathbf{x})\right\rangle
$$

where $\left|\phi_{\nu}(\mathbf{x})\right\rangle$ is a state with definite (classical) amplitudes of certain fundamental fields $\phi_{\nu}(\mathbf{x}), \nu=1,2, \ldots, N$. If $I_{1}$ is some region of space, with $I_{2}$ being its complement, $\left|\phi_{\nu}(\mathbf{x})\right\rangle$ is approximately (neglecting Casimir-type entanglement of the relativistic vacuum) a direct product, $\left|\phi_{\nu}(\mathbf{x})\right\rangle_{I_{1}}\left|\phi_{\nu}(\mathbf{x})\right\rangle_{I_{2}}$. The Schmidt representation with respect to $I_{1}$ and $I_{2}$ reads

$$
|\alpha(t)\rangle=\sum_{l} \hat{c}_{l}\left|\hat{\beta}_{l}(t)\right\rangle_{I_{1}}\left|\hat{\gamma}_{l}(t)\right\rangle_{I_{2}}
$$

where the factor states can again be written as superpositions of field eigenstates, for example

$$
\left|\hat{\beta}_{l}(t)\right\rangle_{I_{1}}=\int_{\left(I_{1}\right)} \mathcal{D} \phi_{\nu}(\mathbf{x}) \hat{\Psi}_{l}\left[\phi_{\nu}(\mathbf{x}), t\right]\left|\phi_{\nu}(\mathbf{x})\right\rangle_{I_{1}}
$$

The functional integral $\int_{\left(I_{1}\right)} \mathcal{D} \phi_{\nu}(\mathbf{x}) \ldots$ now runs over all field amplitudes on the space region $I_{1}$ only. Provided $I_{1}$ carries precisely the ultimate observer system, one might postulate a (partly reversible) branching of consciousness into these product states which are distinguished by their index $l$.

If the region $I_{1}$ shrinks to a point $\mathbf{x}_{0}$, say, the states $\mid \phi_{\nu}(\mathbf{x}\rangle_{I_{1}}$ become

$$
\left|\phi_{\nu}(\mathbf{x})\right\rangle_{\mathbf{x}_{\mathbf{0}}}=\left|\xi_{\nu}\right\rangle:=\left|\phi_{\nu}\left(\mathbf{x}_{\mathbf{0}}\right)\right\rangle
$$


that is, states characterized by the values of all fields at the point $\mathbf{x}_{\mathbf{0}}$. The quantum states of such a point can therefore be written as

$$
|\beta\rangle_{\mathbf{x}_{\mathbf{0}}}=\int d \xi_{1} \ldots d \xi_{N} f\left(\left\{\xi_{\nu}\right\}\right)\left|\left\{\xi_{\nu}\right\}\right\rangle
$$

The manifoldness of these superpositions appears rich enough to represent primitive conscious awareness in a psycho-physical parallelism. It seems that quantum superpositions have never been considered, for example, in neuronal models, since only classical states of definite neuronal excitation are usually taken into account. These quasi-classical states are also measured by external neurobiologists. Quantum theory would admit their superpositions, too, thus giving rise to a far greater variety of physical states which may be experienced by the subjective observer. (Note added: When used for information processing, such superpositions would now be called "quantum bits". As demonstrated by M. Tegmark, they can not be relevant for neuronal and similar processes in the brain - see Phys. Rev E61, 4194 (2000) or quant-ph/9907009.)

However, the physical carrier of states of primitve consciousness can neither be expected to include unconscious memories, nor those neuronal activities which are related to "behavior" (such as speech). Evidently, most brain activities remain unconscious, but nonetheless contribute to the complexity of "conscious behavior". For example, the quantum state on the region $I_{2}$, which by definition is external to the ultimate observer system, may be further divided into the rest of the brain, $I_{21}$, and the external world in the usual sense, $I_{22}$. The states $\left|\hat{\gamma}_{l}\right\rangle_{I_{2}}$ may then again be written as

$$
\left|\hat{\gamma}_{l}\right\rangle_{I_{2}}=\sum_{m} \hat{d}_{m}^{(l)}\left|\hat{\delta}_{m}^{(l)}\right\rangle_{I_{21}}\left|\hat{\epsilon}_{m}^{(l)}\right\rangle_{I_{22}}
$$

The type of states $\left|\hat{\delta}_{m}^{(l)}\right\rangle_{I_{21}}$ is thus essentially determined by the unavoidable and mostly irreversible interaction of the brain with its environment $I_{22}$. The observer system $I_{1}$ - perhaps assumed to interact only with $I_{21}-$ cannot, therefore, observe relative phases between states of different $m$ (see Section 4 of Ref. 38). If the states on $I_{21}$ possess memory properties, which must be "robust" or dynamically stable [14], these properties (in contrast to the states which carry consciousness) would behave classically: memory is irreversibly objectivized by means of the unavoidable interaction with its environment. 


\section{REFERENCES}

1. G. Ludwig, Z. Physik 135, 483 (1953).

2. A. Danieri, A. Loinger, and G. Prosperi, Nucl. Phys. 33, 297 (1962).

3. W. Weidlich, Z. Physik 205, 199 (1967).

4. I. Prigogine and L. Rosenfeld, Nature 240, 25 (1972).

5. 0. Kübler and H. D. Zeh, Ann. Phys. ( N. Y.) 76, 405 (1973).

6. W. Heisenberg, Physik und Philosophie (Hirzel, Stuttgart, 1972).

7. B. d'Espagnat, Conceptual Foundations of Quantum Theory (Benjamin, New York, 1971).

8. D. J. Bohm and B. J. Hiley, Found. Phys. 3, 93 (1975).

9. A. Einstein, N. Rosen, and B. Podolski, Phys. Rev. 47, 777 (1935).

10. D. Bohm and Y. Aharonov, Phys. Rev. 108, 1070 (1957).

11. J. F. Clauser and A. Shimony, Rep. Prog. Phys. 41, 1881 (1978).

12. J. S. Bell, Physics 1, 195 (1964).

13. K. Baumann, Z. Naturforsch. A25, 1954 (1970); Acta Phys. Austr. 36, 1 (1972).

14. H. D. Zeh, Found. Phys. 1, 69 (1970) - reprinted in J.A. Wheeler and W.H. Zurek, Quantum Theory and Measurement (Princeton UP, 1983).

15. H. Everett III, Rev. Mod. Phys. 29, 454 (1957).

16. R. Zwanzig, in Boulder Lectures in Theoretical Physics, Vol. 3 (1960), p. 106.

17. L. Van Hove, Physica 22, 343 (1956).

18. I. Prigogine, Nonequilibrium Statistical Mechanics (New York, 1962).

19. P. N. Argyres and P. L. Kelly, Phys. Rev. 134, A98 (1964).

20. R. M. Lewis, J. Math. Phys. 8, 1448 (1967).

21. A. Wehrl, Rev. Mod. Phys. 50, 221 (1978).

22. R. Mirman, Found. Phys. 5, 491 (1975).

23. L. Szilard, Z. Physik 53, 840 (1929).

24. L. Brillouin, Science and Information Theory (Academic, New York, 1956).

25. H. D. Zeh, in Proc. 49th Enrico Fermi School of Physics, B. d'Espagnat, ed. (Academic, New York, 1972), p. 263.

26. E. P. Wigner, Am. J. Phys. 31, 6 (1963).

27. I. M. Jauch, Foundations of Quantum Mechanics (Addison-Wesley, Reading, Mass., 1968).

28. F. London and E. Bauer, La theorie de I'observation mecanique quantique (Hermann, Paris, 1939).

29. E. P. Wigner, in The Scientist Speculates, L. I. Good, ed. (Heinemann, 
London, 1962).

30. H. D. Zeh, Found. Phys. 5, 371 (1975).

31. Ph. Pearle, Phys. Rev. D13, 857 (1976); Intern. J. Theor. Phys. 18, 489 (1979).

32. D. Bedford and D. Wang, Nuovo Cimento 26B, 313 (1975).

33. C. Misner, K. S. Thorne, and I. A. Wheeler, Gravitation (Freeman, San Francisco, 1973), p. 1217.

34. E. Schmidt, Math. Annalen 63, 433 (1907).

35. E. Schrödinger, Proc. Cambr. Phil. Soc. 31, 555 (1935).

36. H. D. Zeh, in Proc. of the 4th Conference on the Unity of the Sciences (Int. Cultural Foundation, New York, 1975), p. 287.

37. R. W. Sperry, in The Neurosciences Third Study Progrum, F. 0. Schmidt and F. G. Worden, edts. (MIT Press, Cambridge, Mass., 1974).

38. H. D. Zeh, Found. Phys. 3, 109 (1973) - quant-ph/0306151

39. T. Gold, ed., The Nature of Time (Cornell, Ithaca, N. Y., 1967).

40. B. Gal-Or, Modern Developments in Thermodynamics (Wiley, New York, 1974). 